\title{
DOPPLER-RELATED FOCUSING ASPECTS IN THE TOPS IMAGING MODE
}

\author{
Marc Rodriguez-Cassola, Pau Prats-Iraola, Francesco De Zan, Rolf Scheiber, Andreas Reigber
}

Microwaves and Radar Institute, German Aerospace Center (DLR)

\begin{abstract}
The distortions caused in conventionally focused TOPS SAR images which originate from the azimuth-variant Doppler centroid within a burst are presented and analyzed. In particular, the azimuth distortions due to topography mismatch in focusing stages and the range distortions due to the assumption of validity for the stop-and-go approximation are expounded in detail. Compensation strategies to accommodate the two effects in an accurate and precise manner are discussed and validated with TOPS data acquired with TerraSAR-X.
\end{abstract}

\section{INTRODUCTION}

This paper addresses two aspects related to the focusing of SAR raw data acquired in the TOPS (Terrain Observation by Progressive Scans) mode [1]. Due to the TOPS acquisition geometry and the steering in azimuth of the antenna, targets at different azimuth positions within the burst are observed under different squint angles, i.e., there is an azimuth-variant Doppler centroid within the burst. If this fact is not properly considered at focusing stages, then undesired distortions both in the azimuth and range dimensions are introduced. The azimuth distortions are caused by the local mismatch of the topography; range distortions arise from the assumption of validity of the stop-and-go approximation. Conventional spaceborne SAR focusing schemes will be in general unable to provide undistorted TOPS SAR images. These effects are discussed and evaluated for typical TerraSAR-X scenarios. Compensations strategies are presented and validated with TerraSAR-X TOPS data.

\section{AZIMUTH DISTORTIONS DUE TO TOPOGRAPHY MISMATCH}

Due to the curvature of the orbits, targets at the same (beam center) slant ranges but at different heights have slightly different phase histories. In other words, the effective velocity of a given target depends on its height. A common assumption of Fourier-based SAR focusing kernels is the constant effective velocity for a common range bin. This translates into the use of the same effective velocity within a large azimuth block of data, e.g., a whole burst. Since this effective velocity

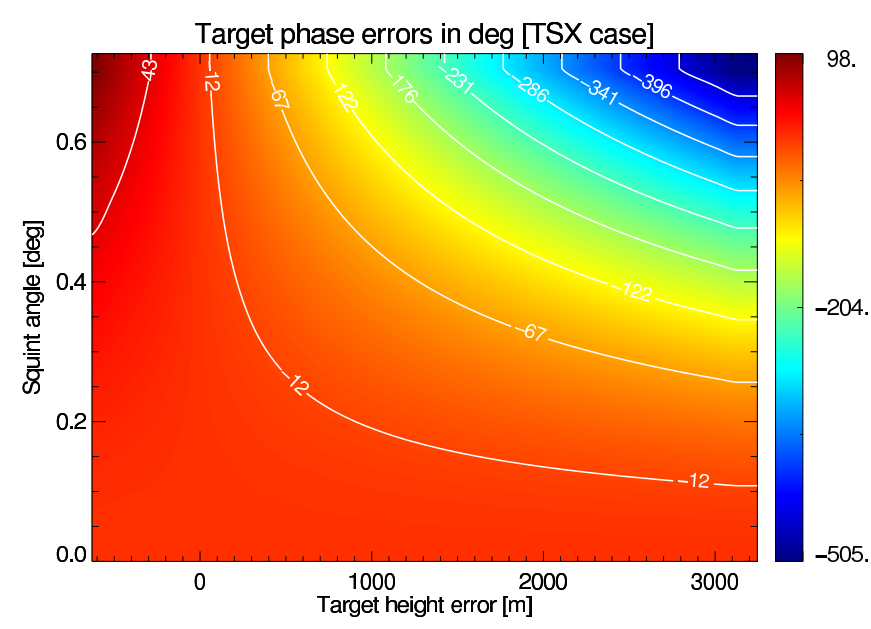

Fig. 1. Phase error of the targets as a function of the squint angle and the topographic height error with respect to the reference (processing) level. A Keplerian TerraSAR-X like orbit has been used in the computations.

is depending on the topography, only an isoline ${ }^{1}$ is perfectly matched, i.e., deviations of the real topography of the scene with respect to the processing isoline results in focusing errors. For the usual low-to-medium resolutions of TOPS systems, this mismatch does not introduce significant defocusing of the impulse response, but it causes a phase offset and a shift of its azimuth position. The phase error of a given target can be expressed as

$$
\delta \phi \approx-\frac{4 \pi}{\lambda} \cdot r_{0} \cdot \frac{\left(\frac{\lambda \cdot f_{\mathrm{DC}}}{2 \cdot v_{\mathrm{e}}}\right)^{2}}{\sqrt{1-\left(\frac{\lambda \cdot f_{\mathrm{DC}}}{2 \cdot v_{\mathrm{e}}}\right)^{2}}} \cdot \frac{\Delta v_{\mathrm{e}}(h)}{v_{\mathrm{e}}}
$$

where $r_{0}$ is the range of the target, $\lambda$ is the wavelength, $f_{\mathrm{DC}}$ is the Doppler centroid with which the target is seen, $v_{\mathrm{e}}$ is the effective velocity used in the processing, and $\Delta v_{\mathrm{e}}$ is the difference between $v_{\mathrm{e}}$ and the real effective velocity of the considered target, which is a function of its height $h$. The

\footnotetext{
${ }^{1}$ Due to the orbit's and Earth's curvature, this line of constant effective velocity shows slight deviations with respect to the constant height contour line.
} 


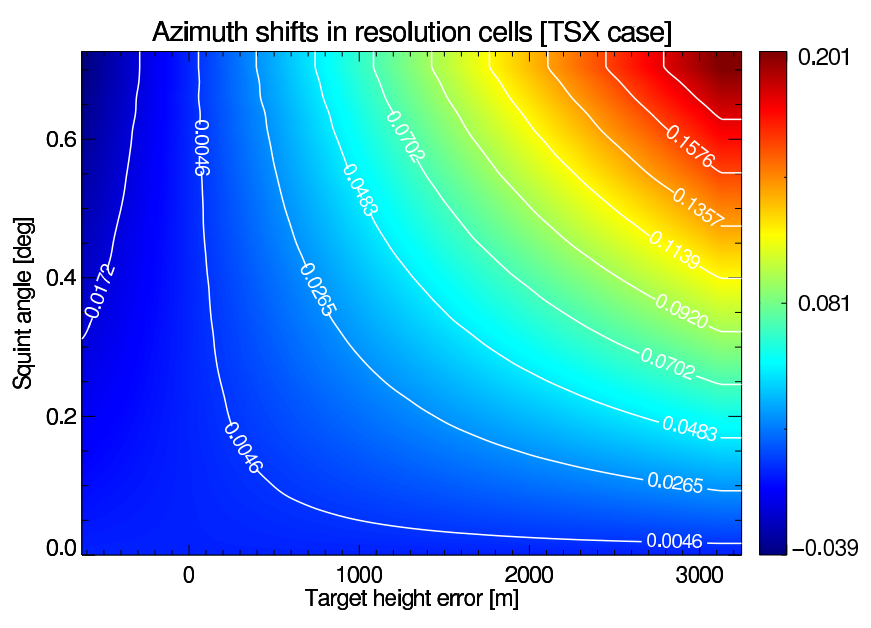

Fig. 2. Azimuth shifts of the focused target as a function of the squint angle and the topographic height difference with respect to the reference (processing) level. Processed bandwidth of $450 \mathrm{~Hz}$.

azimuth shift of the impulse response takes the value [2, 3]

$$
\delta t \approx f_{\mathrm{DC}} \cdot \frac{\Delta K_{\mathrm{a}}}{K_{\mathrm{a}}^{2}} \approx-\lambda \cdot r_{0} \cdot f_{\mathrm{DC}} \cdot \frac{\Delta v_{\mathrm{e}}(h)}{v_{\mathrm{e}}^{3}},
$$

where $K_{\mathrm{a}}$ is the Doppler rate of the target. Note that the induced shift is wavelength-independent, since it cancels out with the product wavelength-Doppler centroid. The term $\Delta v_{\mathrm{e}}(h)$ can be well approximated as a linear function of $h$ whose slope depends on the orbit parameters. For a TerraSAR-X case and a topographic span of $4 \mathrm{~km}, \Delta v_{\mathrm{e}}$ varies about $2 \mathrm{~m} / \mathrm{s}$. Fig. 1 shows the phase error of the focused targets for a typical TerraSAR-X acquisition, with up to 0.7 deg squint angle. The error is displayed as a function of the squint and the topographic height, assuming the reference height used in the processing is $625 \mathrm{~m}$. Significant errors in the phase of the TOPS images are expected for scenes with large topographic variations if the effect is not properly accounted for. Fig. 2 shows the corresponding azimuth shifts (in resolution cells) for the same TerraSAR-X case for a processed azimuth bandwidth of $450 \mathrm{~Hz}$. As in the previous case, significant azimuth shifts of up to 0.25 resolution cells in the TerraSAR-X case can be observed within the TOPS SAR images if topography is not locally accommodated. In the burst overlap areas, the Doppler centroid of the targets has about the same absolute value but an opposite sign for mean zero-squinted acquisitions. By inspecting (1) and (2) we can see that in the burst overlap areas the inter-burst phase error is cancelled, whereas the inter-burst azimuth coregistration error takes the form

$$
\delta t_{\mathrm{burst}}=-\lambda \cdot r_{0} \cdot \Delta f_{\mathrm{ovl}} \cdot \frac{\Delta v_{\mathrm{e}}(h)}{v_{\mathrm{e}}^{3}}
$$

where $\Delta f_{\text {ovl }}$ is the spectral separation between the maximum fore and aft steering angles, which is in the order of $6 \mathrm{kHz}$ for

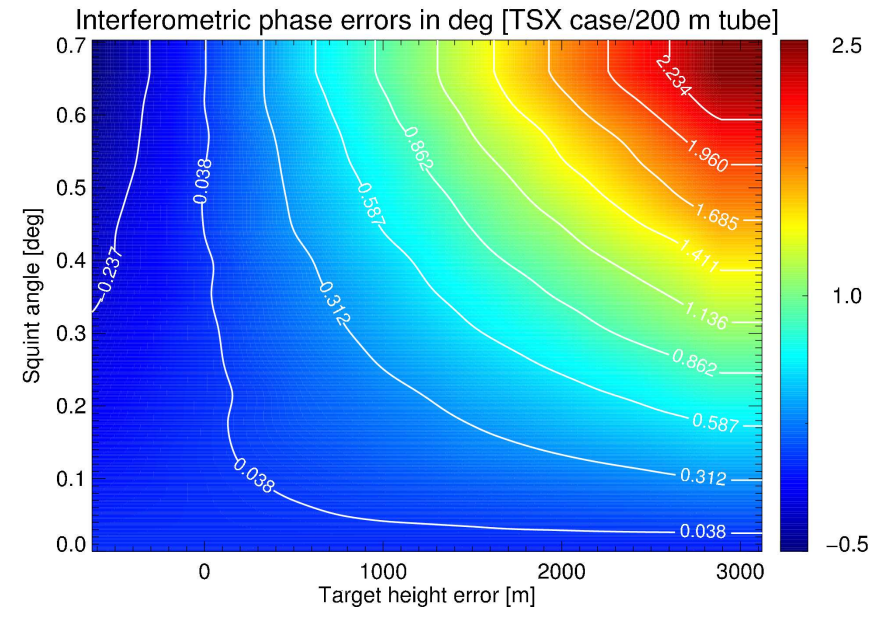

Fig. 3. Interferometric phase errors caused by topography mismatch for the TerraSAR-X case. Orbital tube of $200 \mathrm{~m}$.

\section{TerraSAR-X.}

In interferometric mode, however, things improve due to the similarity of the observed phase and positioning errors for both master and slaves images. In the TerraSAR-X case, with an orbital tube of about $200 \mathrm{~m}$, the differences of the positioning errors are insignificant and the interferometric phase errors remain small. In Fig. 3, we can see that the interferometric errors cancel out for the TerraSAR-X case even in the case of large topographic variations. However, phase and coregistration errors increase under larger baselines or nonparallel orbits.

\subsection{Post-processing algorithm for spaceborne SAR to- pography accommodation (PASTA)}

The post-processing algorithm for spaceborne SAR topography accommodation (acronym PASTA) is a precise yet efficient manner to accommodate topographic variations within range bins. PASTA is capable of achieving excellent results even if the data have been focused neglecting the dependence of the effective velocity with range (e.g., a classical assumption of the canonical omega-K algorithm). PASTA is applied once the burst has been focused and can be straightforwardly parallelized. The block diagram of PASTA is shown in Figure 4. In the diagram, the effective velocity used in the processing, $\tilde{v}_{\mathrm{e}}$, is range-dependent. The real effective velocities of the scene, represented in the matrix $v_{\mathrm{e}}$, is computed using the real DEM of the scene. PASTA works as a post-processing approach, i.e., on the focused SLC. The first step is a deramping to set the data on a common frequency reference. The algorithm works with short blocks in azimuth centred around the pixel to be corrected. An azimuth DFT is computed on the block, after which the compensation phase $\Delta \Phi_{\mathrm{AC}}$ is applied. This residual correction is performed in the range-Doppler domain. After this, the whole azimuth bin is corrected. Once 


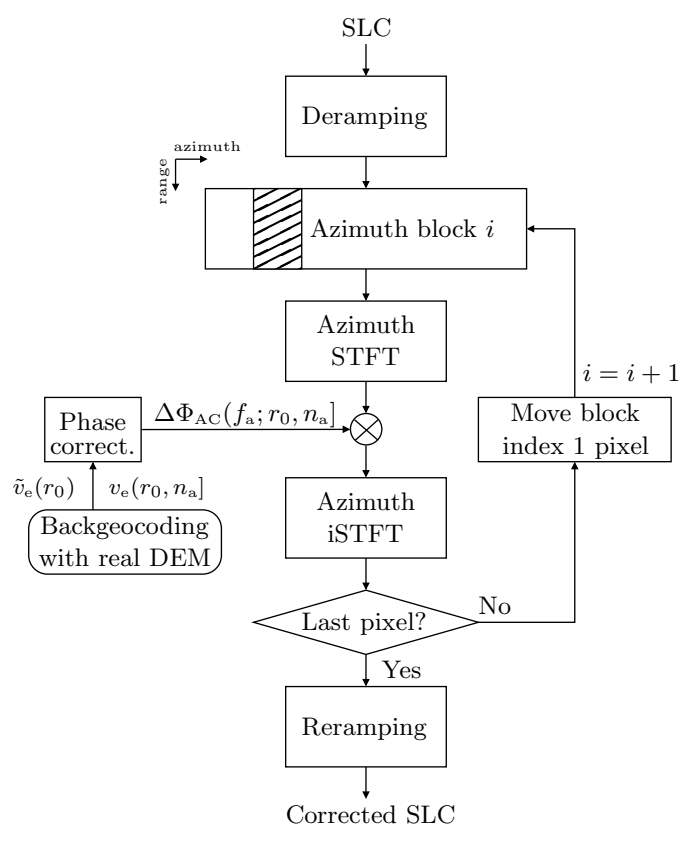

Fig. 4. Block diagram of of the post-processing algorithm for spaceborne SAR topography accommodation (PASTA).
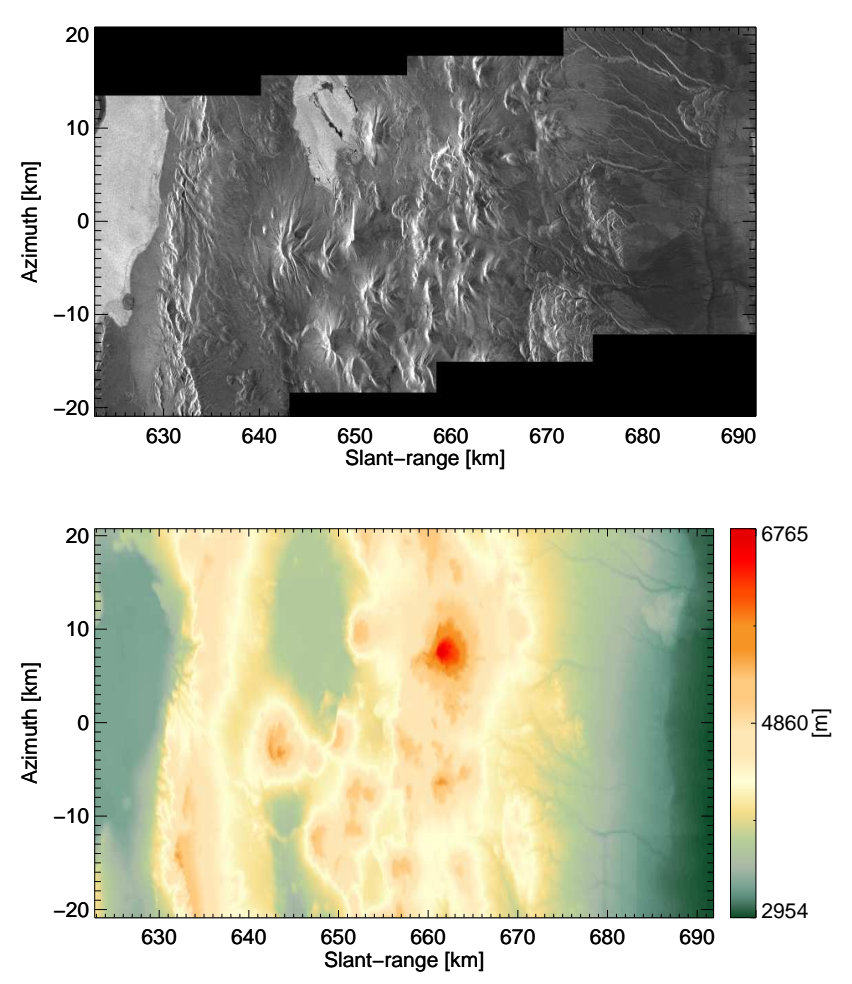

Fig. 5. TerraSAR-X TOPS reflectivity image (3 bursts) over Atacama desert, Chile and the derived DEM (bottom).
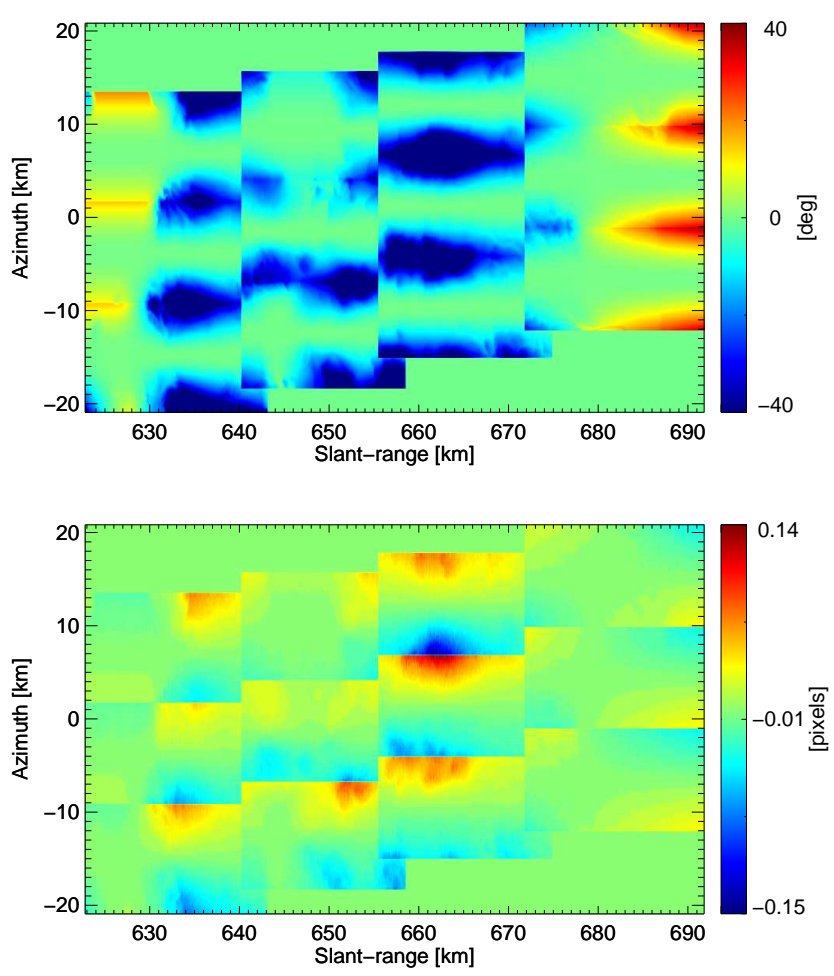

Fig. 6. Phase errors (top) for the reference SLC processed on the ellipsoid before and after PASTA. Corresponding azimuth shfits (bottom).

all pixels are corrected, the data are reramped to their original frequency reference. The algorithm is easily parallelisable and runs in comparable times as the focusing of the burst itself using [4]. Experimental TOPS data acquired by TerraSAR-X over Atacama desert, Chile, have been used to validate the approach. Fig. 5 shows the reflectivity image of the first 4 bursts and the corresponding DEM (bottom) ; note the topographic variations within the scene are over $3500 \mathrm{~m}$.

To better illustrate the results, a processing over an ellipsoidal height of $3700 \mathrm{~m}$ is used as an error reference; PASTA is then applied on the data. ${ }^{2}$ Fig. 6 shows the analysis between the reference image processed on the SLC and the corrected one using PASTA. The top image shows the phase errors and the bottom image shows the azimuth shifts. Both agree with the values obtained in Figs. 1 and 2. Fig. 7 shows the interferometric errors between the interferogram computed with the reference images processed on the ellipsoid, and the interferogram computed with the reference images corrected with PASTA. Values are comparable with those of Fig. 3.

\footnotetext{
${ }^{2} \mathrm{~A}$ more realistic processing takes into account the real DEM of the scene capable of a range-dependent, azimuth-averaged topographic accommodation which might result in smaller errors than the ones presented here.
} 


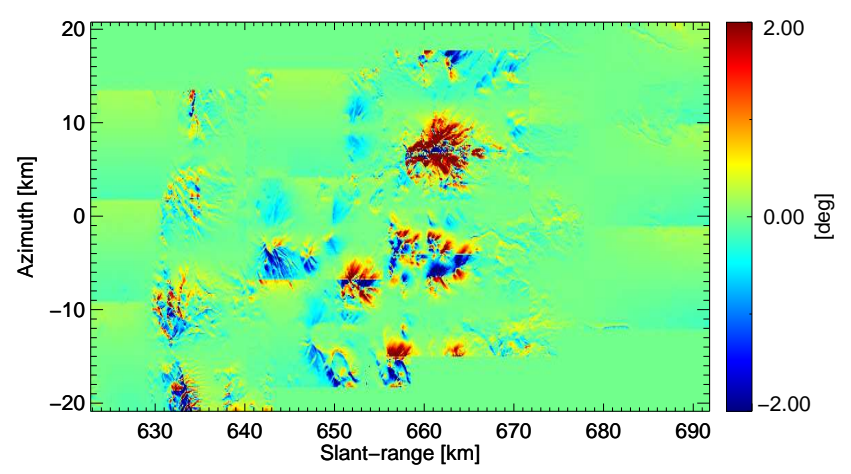

Fig. 7. Interferometric errors for reference SLCs processed on the ellipsoid before and after PASTA.

\section{THE STOP-AND-GO APPROXIMATION IN THE TOPS MODE}

In conventional spaceborne SAR processing, the motion of the platform during the transmission and reception of the chirp signal is usually neglected. This approximation has two effects. A slow-time one caused by the motion of the satellite between transmission and reception [5]; and a fasttime effect dealing with the motion of the satellite during the transmission and reception of the chirp signal itself. As an example, for a chirp signal of a length of about $50 \mu \mathrm{s}$, TerraSAR-X moves about $38 \mathrm{~cm}$ in the azimuth direction. One intuitive way to visualize this is by considering different azimuth-frequency phase ramps for different range frequencies, since there is a direct mapping between fast-time and range frequency. In the frame of pulsed radars, the effect was already discussed in [2], where the analysis was done in terms of a range compression mismatch, and in the context of high-resolution SAR imaging in [6, 7].

In the TOPS mode, the effect corresponds with a shift in the range dimension. Effectively, due to the small azimuth bandwidth, only the phase ramp in range is mainly present, which has an opposite sign for the targets located at the beginning and the end of a TOPS burst. This means that opposite shift is being introduced, hence producing a coregistration error in range at burst edges between adjacent bursts. For the TerraSAR-X case, typical values for this differential range shift can of about 0.3 resolution cells. As the fast-time effect is space-invariant, it can be easily corrected in the 2D frequency domain using the following phase function [6, 7]

$$
H_{\mathrm{ss}}\left(f_{\mathrm{a}}, f_{\mathrm{r}}\right)=\exp \left(\mathrm{j} \cdot 2 \pi \cdot f_{\mathrm{a}} \cdot \frac{f_{\mathrm{r}}}{K_{\mathrm{r}}}\right),
$$

where $K_{\mathrm{r}}$ is the chirp rate, $f_{\mathrm{a}}$ the azimuth frequency, $f_{\mathrm{r}}$ the range frequency. Fig. 8 exemplifies this effect using TerraSAR-X TOPS data, where the impulse response function over a corner reflector $(\mathrm{CR})$ located at the overlap area
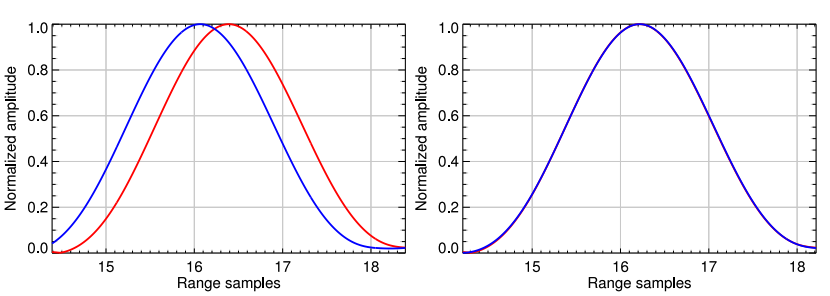

Fig. 8. Interpolated range impulse response of a CR. The CR is located at the overlap region between two bursts. The lines correspond to the (red) first burst and the (blue) following burst. Stop-and-go not corrected (left) and corrected (right).

is shown for the two bursts involved, without and with the proposed correction. Without the correction the shift is of about 0.3 range samples.

\section{SUMMARY}

The paper has presented the intrinsic distortions of TOPS SAR images due to the azimuth dependence of the Doppler centroid within a burst. In particular, distortions in the two image dimensions could be identified: a) in azimuth dimension induced by topography mismatch in focusing stages, and b) in range dimension if the stop-and-go approximation is assumed in the processing. Compensation strategies for both effects have been discussed and validated with real TerraSAR-X TOPS data. Note that the aforementioned aspects also apply to the ScanSAR mode, since a Doppler variation is also present within the burst, although smaller.

\section{REFERENCES}

[1] F. De Zan and A. Monti Guarnieri, "TOPSAR: Terrain observation by progressive scans," IEEE Trans. Geosci. Remote Sens., vol. 44, no. 9, Sept. 2006.

[2] J. C. Curlander and R. N. McDonough, Synthetic Aperture Radar: Systems and Signal Processing, John Wiley \& Sons, New York, USA, 1991.

[3] Pau Prats et al., "TOPS interferometry with TerraSAR-X," IEEE Trans. Geosci. Remote Sens., vol. 50, no. 8, pp. 3179-3188, Aug. 2012.

[4] Pau Prats et al., "Processing of sliding spotlight and TOPS SAR data using baseband azimuth scaling," IEEE Trans. Geosci. Remote Sens., vol. 48, no. 2, Feb. 2010.

[5] Helko Breit et al., "TerraSAR-X SAR processing and products," IEEE Trans. Geosci. Remote Sens., vol. 48, no. 2, Feb. 2010.

[6] Pau Prats-Iraola et al., "High precision SAR focusing of TerraSAR-X experimental staring spotlight data," in Proc. IEEE International Geoscience and Remote Sensing Symposium (IGARSS'12), Munich, Germany, July 23-27 2012.

[7] Pau Prats-Iraola et al., "On the processing of very highresolution spaceborne SAR data," IEEE Trans. Geosci. Remote Sens., in review. 